\title{
Conceptualizing Mental Health Through Bhutanese Refugee Lens: Findings from a Mixed Methods Study
}

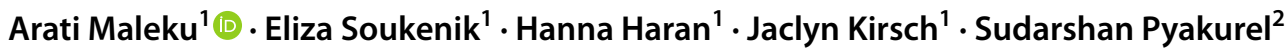

Received: 9 November 2020 / Accepted: 5 May 2021 / Published online: 15 May 2021

(c) The Author(s), under exclusive licence to Springer Science+Business Media, LLC, part of Springer Nature 2021

\begin{abstract}
Despite the rapidly growing need to understand mental health challenges faced by refugee subpopulations, there is a dearth of literature exploring mental health conceptualization through the unique refugee lens. Guided by historical trauma theory, we gathered data using a two-phase explanatory sequential mixed-methods study (quantitative: $n=40$; qualitative: $n=6$ ) in a Midwestern U.S. region to understand mental health conceptualization from the Bhutanese refugee perspective by examining the cultural meaning and perception of mental health, describing experiences of mental health problems, and examining cultural protective factors and coping strategies. We argue that recognition of refugees' conceptualization of mental health and identification of cultural protective factors is paramount to healing. Findings emphasize the need to understand historical and cultural perspectives in cross-cultural contexts for the development and implementation of culturally responsive services. Our study also contributes to emerging knowledge on methodological rigor in research among understudied, hard-to-reach, small populations.
\end{abstract}

Keywords Bhutanese refugees $\cdot$ Mental health $\cdot$ Mixed methods $\cdot$ Historical trauma

\section{Introduction}

Since 2006, more than 86,000 Bhutanese refugees have resettled in the United States (U.S. Department of State, 2017), one of the largest refugee resettlement programs coordinated by the United Nations High Commissioner for Refugees in recent years (Gurung \& Baidya, 2010; Shrestha, 2015). An ethnically and linguistically Nepali minority group called Lhotshampas, Bhutanese refugees were forced

Arati Maleku

maleku.1@osu.edu

Eliza Soukenik

soukenik.3@osu.edu

Hanna Haran

haran.1@osu.edu

Jaclyn Kirsch

kirsch.35@buckeyemail.osu.edu

Sudarshan Pyakurel

spyakurel@bccoh.org

1 College of Social Work, Ohio State University, Columbus, USA

2 Bhutanese Community of Central Ohio, Columbus, USA to flee genocide, torture, and other human rights violations (Tol et al., 2010) in the early 1990s when the Bhutan government enacted policies to exclude and denationalize ethnic and religious minorities (Evans, 2010). The majority of Bhutanese refugees in the United States arrived between 2008 and 2012 (Centers for Disease Control and Prevention, n.d.) after two decades of displacement in refugee camps in neighboring Nepal, which denied them citizenship despite their cultural ties to the country (Chase \& Sapkota, 2017).

Bhutanese refugees have a long and painful history of collective historical trauma from forced displacement, separation from family members, and loss of citizenship that continues to affect them on both individual and community levels in places of resettlement (Rinker \& Khadka, 2018). After their third-country resettlement in the United States, this experience of collective historical trauma is often compounded by post-resettlement challenges including but not limited to adjusting to a new environment, lack of employment and reliable social services, and language barriers, causing Bhutanese refugees to suffer from poor mental health outcomes (Ellis et al., 2016). Mental health is a public health concern among Bhutanese refugees, and these problems are experienced across generations (Rinker \& Khadka, 2018). Bhutanese refugees suffer from alarming rates of 
substance misuse, depression, anxiety, and PTSD (Cochran et al., 2013), the latter three at significantly higher rates than the general U.S. population (Vonnahme et al., 2015). Further, Bhutanese refugees have been found to attempt and complete suicide at high rates-both historically in refugee camps (Schininà et al., 2011) and today in resettlement contexts (Ao et al., 2016). Despite these documented mental health risks, however, mental health service utilization rates remain low among Bhutanese refugees due to direct and indirect barriers at the individual and system levels (Wylie et al., 2018): cultural barriers, acculturation stressors, economic barriers, language barriers, gaps in culturally responsive services, and the larger political environment of visible minority status (Adhikari et al., 2015; Derr, 2016; Van Ommeren et al., 2001). Further, a deeper understanding of mental health from the unique Bhutanese refugee lens also remains limited (Vonnahme et al., 2015), largely due to Westernized approaches to mental health care that often overlook refugees' experiences and cultural explanations of symptoms and distress (Im et al., 2017).

To effectively address community mental health needs and develop culturally responsive interventions, a culturally grounded understanding of mental health, including the meaning of mental health, experience of distress, and cultural protective factors such as community coping strategies, is crucial. Studies that conceptualize mental health from community-grounded perspectives are warranted to devise culturally tailored programs and services that can mitigate mental health challenges and empower these new members of American society (Im et al., 2017). In this study, we sought to provide a comprehensive understanding of mental health through the Bhutanese refugee lens to provide new directions and insights for eliminating mental health inequities in this population. We begin with a general review of refugee mental health and mental health services, with particular attention to Bhutanese refugees resettled in the United States, to set the context of the study.

\section{Bhutanese Refugees, Mental Health, and Mental Health Services}

The literature has established that refugees experience trauma before relocation and are particularly vulnerable to stressors upon resettlement (Yakushko et al., 2008). Cumulative migration stressors-premigration trauma, conditions in refugee camps during prolonged periods of transition, and postmigration stressors-contribute to high mental health risks among refugees. Bhutanese refugees' history of collective trauma has been linked with forced displacement from Bhutan, torture, separation from family members, two decades in refugee camps in Nepal, and loss of citizenship that continue to affect them at individual and community levels (Rinker \& Khadka, 2018). Common postmigration difficulties such as language barriers, worries about family back home, separation from family, lack of social support, financial difficulties, difficulty maintaining cultural and religious traditions, employment difficulties, poor access to health care, insufficient help from charities or other agencies, insufficient help from the government, increased family conflict, and lack of community structures for resolving family disputes have been found to be risk factors among Bhutanese refugees (Hagaman et al., 2016). Acculturation stressors among older Bhutanese refugees were associated with cultural differences in values and norms, abrupt changes in living environments, and drastic shifts in ways of living (Im \& Neff, 2020). Although postmigration stressors vary across demographic groups, postmigration stress such as demands of acculturation, language difficulties, decreased support systems, increased caregiving responsibilities, socioeconomic disadvantages, lack of access to services, changes in family dynamics, financial hardship, discrimination, and limited technology skills were found to be major risk factors for depression and other mental health problems among Bhutanese youth and older adults (Poudel-Tandukar et al., 2019). The traditional approach to health care among Bhutanese refugees often relies on home remedies, and medical treatment is only sought when conditions are not resolved, presenting a potential challenge to U.S. health care provision (Maack \& Willborn, 2018). This challenge can be exacerbated by the possible lack of insurance after resettlement benefits expire (Maack \& Willborn, 2018). The lack of help-seeking behavior in the Bhutanese population may also highlight a gap between the need for mental health services and their use, as well as the availability, accessibility, and perceived efficacy of such services (Hagaman et al., 2016). Further, lower mental health service utilization rates among refugees are linked to social, linguistic, and economic factors; clinical severity; cultural differences in symptom presentation; and systemic discrimination (Derr, 2016; van der Boor \& White, 2020).

\section{Culture, Language, and Somatization}

Culture influences how people understand and make meaning of mental health, and exploring cultural dimensions of mental health behaviors necessitates qualitative modes of inquiry to more actively "engage" culturally embedded points of view (Staples \& Widger, 2012). Among refugees, stigma, which shapes negative perceptions of mental illness and psychological treatment, and the fear of bringing shame to themselves as individuals and to their family are key reasons why they do not seek mental health services (MacDowell et al., 2020; Poudel-Tandukar et al., 2019). Further, language barriers in a health care context can lead to fear and uncertainty regarding health outcomes, especially when interpreters are not available for important steps such 
as scheduling appointments or filling out paperwork (van der Boor \& White, 2020). When interpreters are offered (e.g., for meetings with a provider), concerns arise over the translation's accuracy and completeness, interpersonal dynamics, and the insertion of unsolicited personal attitudes or advice (van der Boor \& White, 2020). At psychiatric care centers, language barriers and lack of interpreters can also lead to inappropriate treatment and reduced access to care (Gong et al., 1991). Further, somatization-the expression of physical symptoms of psychological distress, which cannot be explained by medical examination-is widespread among refugees, especially those from non-Western countries (Rohlof et al., 2014). Although there is strong evidence for somatization generally, this phenomenon needs more exploration among immigrants and refugees (Lanzara et al., 2019; Ritsner et al., 2000), including Bhutanese refugees. Studies involving Nepali-speaking populations can provide important insights into the impact of increased somatization in the presentation of psychiatric symptoms, which could be relevant to the Nepali-speaking Bhutanese population (Kohrt et al., 2005). Although psychological trauma is not a unitary construct and has no single linguistic corollary in Nepal (Kohrt \& Hruschka, 2010), the psychological sequelae of negative life events expressed through idioms of distress provide direction regarding somatization of mental health (Kohrt \& Hruschka, 2010). Studies among Bhutanese refugees and the Nepali population in Nepal showed that idioms of psychological trauma and distress related to the impact on the heart-mind, brain-mind, body, spirit, and social status (Kohrt \& Hruschka, 2010). Idioms related to brain-mind dysfunction contributed to mental health stigma, and seeking treatment for heart-mind distress seemed more socially accepted (Kohrt \& Hruschka, 2010). Evidence for "Nepali-Bhutanese syndrome," the term for somatization in this population, is still scant and remains largely anecdotal in the United States (Tolan, 2016).

\section{Economic and Social Stressors}

Although successful adaptation among refugees has been identified by characteristics such as adequate housing, language skills, financial security, and employment, employment has been deemed the most important factor for successful refugee adaptation (Porter \& Haslam, 2003). Economic stress has been shown to be the most salient stressor that stems from difficulty finding employment and opportunities, largely because refugees arrive with premigration challenges in resettlement spaces with underdeveloped networks and environments that often stigmatize refugee status (Baranik et al., 2018). Economic stressors, including cost, time off work, and childcare, compound the difficulties of navigating structural barriers to mental health treatment (van der Boor \& White, 2020). Overall, lack of financial capital is an obstacle for obtaining services, which further limits health insurance access and awareness regarding alternative medical coverage. In the Bhutanese refugee population, Betancourt et al. (2015) found overwhelming concern related to economic hardship — not having enough money for food or rent-and acculturative stressors including language barriers, particularly related to parents and children being unable to communicate with teachers and other school personnel and children struggling to do their homework. Financial stressors and social stressors that cause disruption of family life among Bhutanese refugees were found to lead to increased risk of depression and suicidal ideation (Vonnahme et al., 2015).

\section{Service Barriers}

Mental health professionals are challenged with providing culturally responsive services because of significant gaps in their understanding of the cultural belief systems of subpopulations (Maleku \& Aguirre, 2014, 2018). Although unique historical and contextual experiences generate culturally specific risk and protective factors for mental health, conceptual models of mental health, suicide, and interventions still rely heavily on Western assumptions and understandings (Cramer \& Kapusta, 2017) or reduce refugees to a cultural monolith. Refugees also experience discrimination from providers and staff members, which diminishes the likelihood they will seek additional services (Teunissen et al., 2014). Thus, little is known about mental health challenges in refugee subpopulations. Given the diversity among these subpopulations, one-size-fits-all mental health interventions do not meet their needs and capacities. In-depth exploration using unique refugee perspectives is much needed to establish the scope of the mental health problem in this population and to make subpopulation groups more visible. Exploring local conceptualizations of mental health, culturally appropriate language can facilitate understanding of how populations survive collective trauma and rebuild identity that acknowledges past suffering and loss (Shannon et al., 2015b). This is crucial to unmasking discrepancies and devising tailored programs and services that bolster efforts toward eliminating mental health inequities and encouraging refugee inclusion.

\section{Our Study}

Despite Bhutanese refugees' experience with collective trauma and documented high rates of psychiatric morbidity, disability, and suicide, there is limited research exploring the conceptualization of mental health from the Bhutanese refugee lens (Shannon et al., 2015b; Vonnahme et al., 2013). A culturally rich description of mental health sensemaking from the Bhutanese refugee lens has the potential to inform 
culturally responsive approaches to promoting exchanges of knowledge and expertise to build collaborative care and community partnership and bridge the evident gaps between mental health care and refugee resettlement services. In this study, we sought to broaden our understanding of mental health in the Bhutanese refugee context by: (a) examining the cultural meaning and perception of mental health; (b) describing experiences of mental health problems; and (c) examining cultural coping strategies and cultural protective factors. This comprehensive understanding of mental health conceptualization from the Bhutanese refugee lens will garner new directions and insights for eliminating mental health inequities in this population.

\section{Study Setting}

The Midwestern U.S. region, the setting of our study, ranks among the top five refugee resettlement sites in the country (Refugee Processing Center, 2020). This region hosts the largest Bhutanese refugee population-approximately 23,500 Bhutanese refugees-and this number is expected to increase in the next 5 years (Adhikari et al., 2015). A recent study in the region suggested alarming rates of anxiety symptoms, PTSD, depression, suicide, and substance misuse among resettled Bhutanese refugees in the region (Adhikari et al., 2015). Given the increasing demographic shifts in the region, mental health services are highly uneven and human services organizations face persistent challenges in providing culturally responsive services (Maleku et al., 2018).

\section{Theoretical Framework: Historical Trauma Theory}

History provides groups not only with a platform for mutual affinity, but also with a sense of collective meaning-making, experiences, and identities. As a guiding theoretical framework for our study, we used historical trauma theory, which examines the collective trauma experienced by a population and the effects of repeated traumatic experience across generations (Sotero, 2006). The literature contends that the transmission of collective trauma exists in cultural rituals and artifacts, community events and commemorations, and family narratives diffused through social learning, social identity, and psychodynamics (Tcholakian et al., 2019). The experience of historical trauma in a population has been identified as a potential cause for long-term distress and substance abuse among communities (Ehlers et al., 2013). Given the painful collective historical experience among Bhutanese refugees, understanding mental health through a historical trauma lens might garner new insights on their shared understanding and culturally grounded conceptualization of mental health. Historical trauma theory has been used widely to examine disparate outcomes among
African American and Native American populations (Sotero, 2006) but has seen limited use regarding intergenerational trauma among refugee populations, a phenomenon that is little understood (Sangalang \& Vang, 2017). Populations who faced mass trauma exhibit disease prevalence, psychological distress, maladaptive coping mechanisms, and substance misuse at higher rates-termed historical trauma response-because of trauma's lingering effects, passed down through myriad social and environmental pathways (Heart, 2003). Socially, difficulty with parenting and family relationships and poor communication may lead to psychological distress among children (Sangalang \& Vang, 2017). Environmentally, cultural loss, severed ethnic ties, and a breakdown in community structure can perpetuate trauma in refugee populations and alter how they experience new spaces and realities (Sotero, 2006). Studies have found that refugees situate their traumatic experiences in the political context that led to the violence and disruption of their lives (Shannon et al., 2015a). These compounding pathways for transmission may contribute to higher psychological distress rates among populations with past collective trauma. Therefore, understanding psychological distress among refugees through a historical trauma lens will help articulate how a community makes sense of mental health for that population. This shared understanding could aid providers in implementing culturally responsive services and challenge Western approaches to mitigating mental health experiences by pathologizing or managing symptoms (Tol et al., 2010).

\section{Method}

\section{Research Design}

We used a mixed-methods research design to generate metainference (Creamer, 2018)—which links, compares, contrasts, or modifies inferences generated by quantitative and qualitative strands (Teddlie \& Tashakkori, 2009, p. 300) — to gain a comprehensive understanding of the conceptualization of mental health among Bhutanese refugees. Given the descriptive purpose of our study, we used a two-phase explanatory sequential mixed-methods design for a general understanding of the research problem (Ivankova et al., 2006; Fig. 1). Driven by a quantitative approach and followed by a consecutive qualitative approach, an explanatory sequential mixed-methods design is best suited for studies with a descriptive purpose, wherein the quantitative phase is often abstract and decontextualized and needs a qualitative approach to not only explain or elaborate the quantitative findings, but also provide a richer and more contextualized explanation of the study conditions and results (Watkins \& Gioia, 2015). As such, Phase I of the study included in-person quantitative data collection that gathered broad 


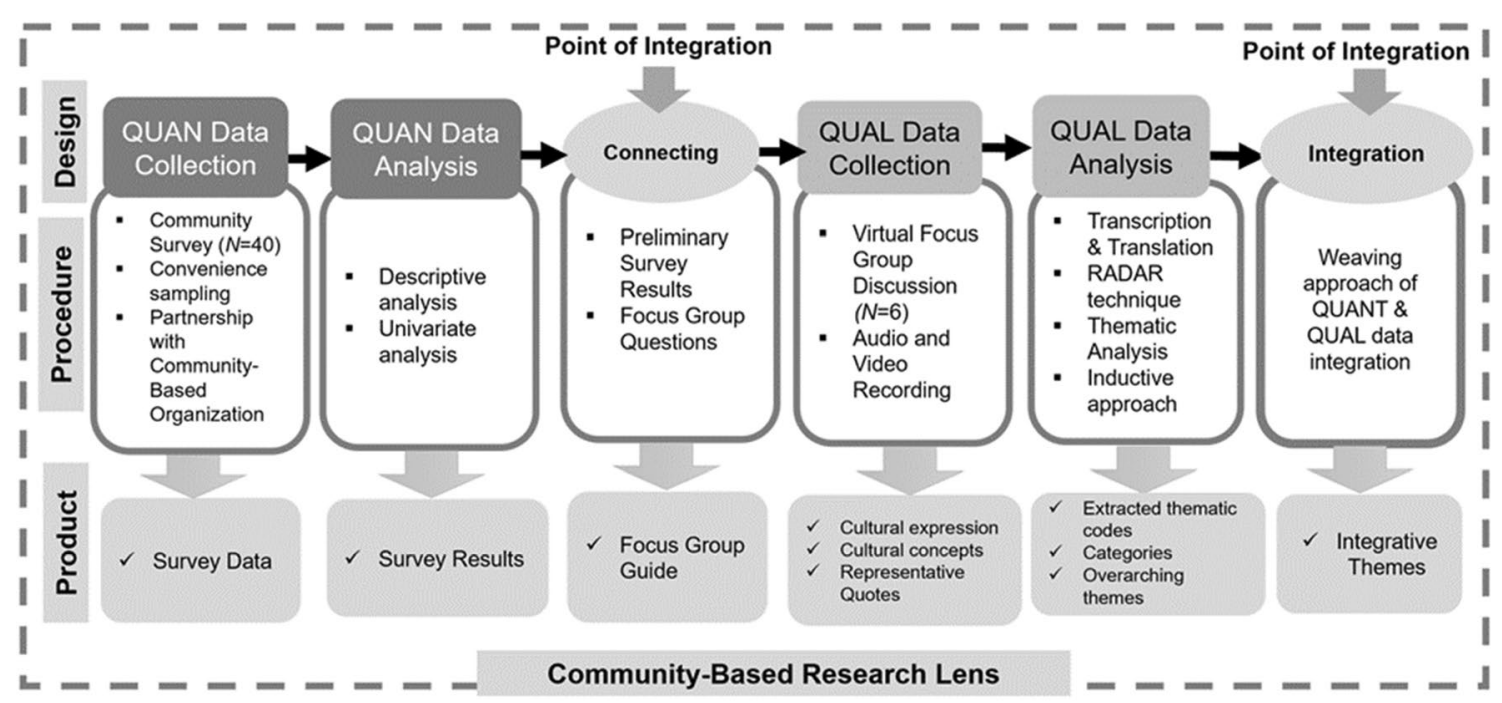

Fig. 1 Procedural diagram: explanatory sequential mixed methods research design

information regarding understanding of mental health and inequities among Bhutanese refugees. Guided by the preliminary findings from the quantitative data regarding patterns and prevalence of mental health, qualitative data collected in Phase II through a virtual focus group discussion (FGD), adjusted due to COVID-19 disruptions, helped explain the quantitative findings and provided in-depth understanding of mental health conceptualization through the Bhutanese refugee lens. Data collection was conducted from February through April 2020. Institutional review board approval was received at the study site.

\section{Sample Recruitment and Data Collection}

The two-phase data collection was conducted in collaboration with a local community-based ethnic organization serving the Bhutanese community, which was the key recruitment site for data collection. Convenience sampling through partnership with community-based organizations presents pragmatic research advantages by providing access to otherwise hard-to-reach groups (Bonevski et al., 2014). We used parallel samples (Onwuegbuzie \& Collins, 2007), which is a sampling strategy in mixed-methods research that specifies that the samples for quantitative and qualitative components are different but drawn from the same population of interest. Parallel sampling was used to seek convergent and divergent themes by purposefully centering diverse community voices to promote interpretive consistency of quantitative and qualitative data (Collins et al., 2007).

The discussion of rigor and practicality related to research with hard-to-reach population should not be overlooked. Sample recruitment among underserved and hard-to-reach populations is seldom straightforward, where data collection procedures and sample size can differ based on population accessibility and study area (Bonevski et al., 2014; Crosby et al., 2010). Therefore, the practicality of recruiting a hardto-reach population sample should not preclude expectations of methodological rigor, often developed in the context of more accessible populations with socially acceptable areas (Crosby et al., 2010). It might seem questionable that as the largest refugee population in the Midwestern region, Bhutanese refugees might not meet the parameters of what is usually considered hard to reach. It is noteworthy, however, that the definition of a hard-to-reach population is not only based on accessibility, geography, and transience, but also on social disadvantages (Bonevski et al., 2014; Shaghaghi et al., 2011). Given the forced displacement context and experiences of structural disadvantages and inequities, the Bhutanese refugee population is a systematically hard-toreach, underserved population (Shaghaghi et al., 2011). The sensitivity of the issue of mental health further compounds the challenges of engaging this population in research. Because research grounded in community perspectives aims to identify the unique experiences of resource-strapped populations (Bonevski et al., 2014), these voices still have the potential to address the paucity of knowledge even with a relatively smaller sample size. These community voices warrant attention and therefore, should not be excluded (Crosby et al., 2010).

We employed multiple methodological strategies to optimize both the research rigor and value of our study findings. The purposeful use of a mixed-methods approach, wherein qualitative data through an FGD provided an opportunity to explain the survey findings grounded in community perspectives and helped broaden the overall descriptive results from the quantitative survey. Studies have suggested including 
three to 12 FGD participants, depending on the nature of the topic area (Padgett, 2017), with as few as four recommended for in-depth discussions on sensitive issues such as mental health (Adler \& Clark, 2015). Our sample size of six FGD participants met the recommended number for an FGD focused on a sensitive topic of mental health. Despite the homogeneity of backgrounds among participants, FGD composition included diverse perspectives based on demographic strata, such as age, gender, and education level, which is a recommended practice to bolster the credibility of focus group data (Hennink et al., 2019). Partnership with the Bhutanese community organization throughout the research process including translation of survey instrument in Nepali using local concepts to capture the cultural vernacular and idioms; participant recruitment and data collection support, which are crucial components of a community-engaged study (Hacker, 2013), facilitated seamless study implementation. Further, member checking and community consultation were utilized to disseminate findings and solicit feedback from the Bhutanese community organization, which helped build population relevance, acceptability, and usefulness of rigorous research data.

\section{Phase I}

Data collection in Phase I included an in-person quantitative paper-based community survey using a 30 -item survey instrument self-administered both in English and Nepali, based on English language proficiency and preference of research participants $(N=40)$. Translators and data collectors from the Bhutanese community organization were used to facilitate data collection, as needed. Each participant received a \$10 USD gift card as an incentive for their time. The 30-item survey instrument included questions covering five major domains: perceptions of mental health; experience with mental health problems; cultural coping strategies and support seeking behavior; mental health services; and an open-ended question regarding mental health in the Bhutanese refugee community. Nominal categories were used to ascertain patterns of mental health meaning and understanding, community perceptions of mental health, cultural stigma associated with mental health, experiences with mental health problems, and perceptions of mental health services. The 30-item survey included standardized scales-namely, the Generalized Anxiety Disorder (GAD7; Spitzer et al., 2006) and the Patient Health Questionnaire (PHQ-9; Kroenke et al., 2001) — to gauge patterns of anxiety and depression symptoms, respectively, as part of the mental health experience domain. GAD-7 is measured with a 4-point scale ranging from 0 (not at all) to 3 (nearly every day) and indicates different levels of anxious symptoms: minimal or none (0-4), mild (5-9), moderate (10-14), or severe ( $\geq 15$; Spitzer et al., 2006). PHQ-9, measured by response options of 0 (not at all), 1 (several days), 2 (more than half the days), and 3 (nearly every day), indicates different levels of depressive symptoms: minimal or none (0-4), mild (5-9), moderate (10-14), or severe ( $\geq 15$; Kroenke et al., 2001). The GAD-7 and PHQ-9 scales have been used among refugee populations as screening instruments to measure psychological distress and identify severity of symptoms (Bjarta et al., 2018). The PHQ-9 scale has been culturally adapted by Kohrt et al. (2016) with lay Nepali terminology and validated among Nepali-speaking populations with high reliability (Cronbach's $\alpha=0.84$ ), moderate internal consistency $(0.54-0.86)$, and high sensitivity and diagnostic odds $(\mathrm{OR}=62.55)$. Similarly, GAD-7 is a widely validated scale in low-income countries, including South Asia, and has been used in Nepal in other recent studies (Gupta et al., 2020). Given the importance of informal care practiced by friends, relatives, and neighbors in the prevention and alleviation of mental distress in the Bhutanese refugee population (Chase \& Sapkota, 2017), we also used the 11-item support seeking domain of the Coping Strategy Indicator Scale (CSI; Desmond et al., 2006) to focus on the mechanisms of coping and support seeking measured by using a 3-point Likert scale $(0=$ not at all, $1=a$ little, $2=a$ lot) as part of the 30 -item survey instrument.

\section{Phase II}

Data collection in Phase II included one 90-min virtual FGD, using a secure Zoom platform to garner in-depth information from Bhutanese key informants $(N=6)$. The virtual FGD had to be adapted due to the disruption caused by COVID-19. The study team, composed of two bilingual (English-Nepali) researchers, conducted the FGD primarily in English, with opportunities for participants to express cultural terminologies in Nepali. Using an FGD guide, researchers asked participants to provide deeper insights on their understanding of mental health, experience of mental health issues, and cultural coping mechanisms and protective factors in the Bhutanese refugee population to further explain and expand the preliminary survey findings in the Bhutanese historical and cultural contexts. Unscripted prompts were used to elaborate on specific mental health topics faced by the Bhutanese community. The FGD was video recorded and transcribed verbatim by the two-person research team. General observatory field notes were also taken by the research team during the FGD. Each participant received a $\$ 25$ e-gift card upon completion of the FGD.

\section{Data Analysis}

Quantitative data from the community survey $(n=40)$ and qualitative data from the FGD $(n=6)$ were first analyzed separately and then integrated to provide a comprehensive 
understanding of mental health conceptualization grounded in the Bhutanese lens. Univariate analyses were used to describe the data and find patterns in the quantitative data collected using SPSS v.25. Composite mean scores were used to calculate the cutoff point for anxiety (GAD-7) and depression (PHQ-9). Mean scores were calculated for CSI to identify mechanisms of support seeking behavior. Reliability measures (Cronbach's $\alpha$ ) showed high levels of internal consistency (GAD-7: $\alpha=0.91$; PHQ-9: $\alpha=0.93$; CSI: $\alpha=0.93)$. We analyzed qualitative data in two stages. First, we used the rapid and rigorous qualitative data analysis (RADaR) technique - an individual and team-based approach to streamlining coding and qualitative data analysis in a systematic way - to develop data reduction tables in Excel (Watkins, 2017). Second, we used thematic coding as the first-cycle coding method (Saldana, 2016) to categorize codes and generate themes based on relationships between codes, frequencies, and meaning across codes. Second-cycle coding methods included axial coding and theoretical coding, wherein the overarching themes were coded, sorted, and synthesized (Saldana, 2016). To maintain methodological rigor in the data analysis, the first and second authors coded the data independently, generated tables with initial and focused codes, and translated the codes into categories, themes, and subthemes. We ran kappa analysis (Cohen's $k$ ) to determine the level of intercoder agreement (McHugh, 2012); results show substantial agreement in data coding and analysis $(k=0.692, p=0.001)$. All authors then re-evaluated data analysis, unanimously agreed on the study's overarching themes, and finalized the translation of themes table (Table 1). Four interrelated overarching thematic clusters emerged from the data. The two databases were then merged for analysis to highlight convergent and divergent themes to present the overall mixed-methods results, a crucial component of a mixed-methods study (Creswell, 2015).

\section{Results: Integration of Quantitative and Qualitative Findings}

Demographic characteristics of the study sample $(N=46)$ are presented in Table 2 . Almost $57.50 \%$ of participants were between 20-30 years of age; median annual income was $\$ 31,000-\$ 40,000 ; 55 \%$ of study participants identified as male and $45 \%$ identified as female; $52.5 \%$ were employed and $45 \%$ were unemployed; and a majority practiced Hinduism $(65 \%)$. Approximately $60 \%$ of survey respondents had been in the United States for ten or more years. Among the key informants $(N=6)$ who participated in the FGD, the mean age was about 32 years, ranging from 20 to 50 years. There was an equal distribution of male and female participants; mean length of stay in [state blinded for review] was about 8 years. Most FGD participants were employed full-time and included a social worker, caseworker, manager, director, and student.

Given the rich information garnered from the FGD to corroborate the descriptive quantitative results, we purposefully established qualitative data as the priority. Then, using a weaving technique, which is a process of integrating both qualitative and quantitative findings on a theme-by-theme or concept-by-concept basis (Fetters et al., 2013), we integrated descriptive and narrative data from quantitative and qualitative phases in four overarching interrelated thematic clusters that emerged from the qualitative data analysis.

\section{Theme 1: Community Understanding of Mental Health}

Findings show that community understanding of mental health in the Bhutanese refugee community is still evolving and rather mixed. Although almost $97.5 \%$ of survey respondents reported that mental health is important to them, participants also shared that there is a persistent lack of awareness due to cultural disbelief in mental health. Survey respondents and discursive FGD discussion highlighted that younger Bhutanese members were more aware and understanding of mental health issues. Recognition and acceptance of mental health in this group were considered to have positive ripple effects in the larger community:

People do have mental health issues, but there is lack of awareness. They are not open to discussing these issues. I feel that more young Bhutanese members are starting to be aware of mental health issues-it's progressing. (Survey participant).

When asked about what mental health meant to the Bhutanese community, survey respondents associated the meaning of mental health (Fig. 2) with five major domains: emotional wellness $(87.5 \%)$, social wellness $(82.5 \%)$, physical wellness $(67.5 \%)$, spiritual wellness $(60 \%)$, and clinical characteristic $(42.5 \%)$.

Although mental health was associated with emotional (bhawanatmak), social (samajik), physical (sharirik), spiritual (aadhyatmik), and clinical (chikitsakiya) attributes, FGD participants highlighted that there is no common understanding of mental health in the Bhutanese population. Participants stated that because people in the Bhutanese community have experienced mental health symptoms for so long, they view them as a part of their daily lives:

The whole idea of mental health is very loosely defined in our community. So, there's like not really [a clear idea]. We still haven't grasped the concept of what actual mental health looks like ... and we don't 


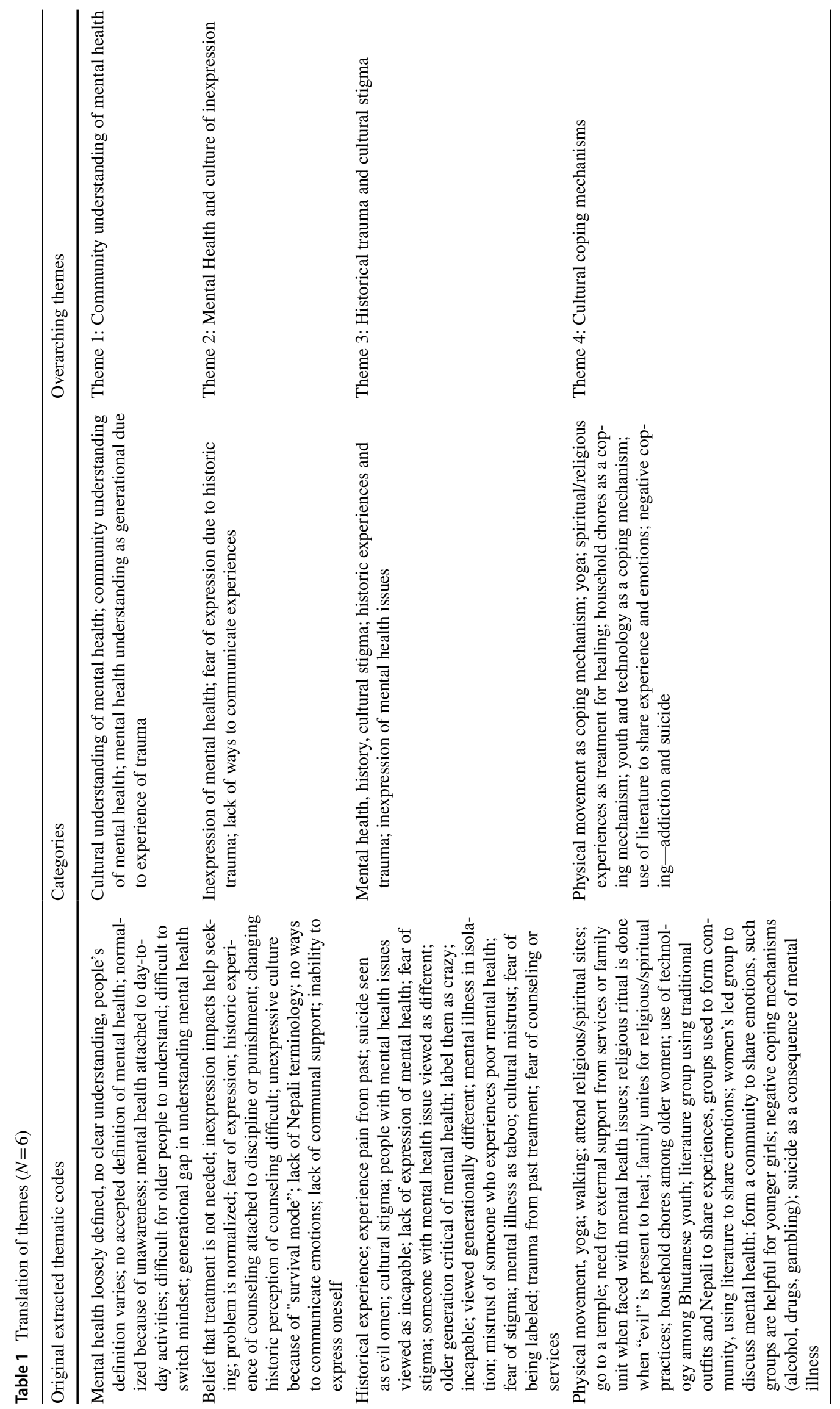


Table 2 Demographic characteristics of study participants $(N=46)$

\begin{tabular}{|c|c|c|}
\hline & $\begin{array}{l}\text { Survey participants } \\
(N=40) \\
f(\%)\end{array}$ & $\begin{array}{l}\text { Focus group participants }(N=6) \\
f(\%)\end{array}$ \\
\hline \multicolumn{3}{|l|}{ Age } \\
\hline Between 20 and 30 years & $23(57.5)$ & Mean $=31.75$ years, $\mathrm{SD}=12.5$ \\
\hline Between 31 and 40 years & $7(17.5)$ & \\
\hline Between 41 and 50 years & $8(20)$ & \\
\hline Between 51 and 60 years & $2(5)$ & \\
\hline \multicolumn{3}{|l|}{ Sex } \\
\hline Male & $22(55)$ & $3(50)$ \\
\hline Female & $18(45)$ & $3(50)$ \\
\hline \multicolumn{3}{|l|}{ Religion } \\
\hline Hinduism & $26(65)$ & \\
\hline Christianity & $8(20)$ & \\
\hline Buddhism & $1(2.5)$ & \\
\hline Other & $5(12.5)$ & \\
\hline \multicolumn{3}{|l|}{ Marital status } \\
\hline Single & $23(57.5)$ & \\
\hline Married & $13(32.5)$ & \\
\hline Separated & $1(2.5)$ & \\
\hline Divorced & $2(5)$ & \\
\hline Missing & $1(2.5)$ & \\
\hline \multicolumn{3}{|l|}{ Employment status } \\
\hline Employed & $21(52.5)$ & \\
\hline Unemployed & $18(45)$ & \\
\hline Missing & $1(2.5)$ & \\
\hline \multicolumn{3}{|l|}{ Annual family income } \\
\hline Less than $\$ 10,000$ & $7(17.5)$ & \\
\hline Between $\$ 10,000$ and $\$ 20,000$ & $2(5)$ & \\
\hline Between $\$ 21,000$ and $\$ 30,000$ & $3(7.5)$ & \\
\hline Between $\$ 31,000$ and $\$ 40,000$ & $11(27.5)$ & \\
\hline Between $\$ 41,000$ and $\$ 50,000$ & $4(10)$ & \\
\hline Between $\$ 51,000$ and $\$ 60,000$ & $4(10)$ & \\
\hline Above 61,000 & $4(10)$ & \\
\hline Missing & $5(12.5)$ & \\
\hline \multicolumn{3}{|l|}{ Place of birth } \\
\hline Bhutan & $24(60)$ & $3(50)$ \\
\hline Nepal & $15(37.5)$ & $3(50)$ \\
\hline Other & $1(2.5)$ & \\
\hline \multicolumn{3}{|l|}{ Education } \\
\hline High school diploma or equivalent & $13(32.5)$ & $2(33.3)$ \\
\hline Some college, but no degree & $6(15)$ & \\
\hline Associate's degree & $3(7.5)$ & \\
\hline Bachelor's degree & $9(22.5)$ & \\
\hline Master's degree & $3(7.5)$ & $2(33.3)$ \\
\hline Never attended school & $1(2.5)$ & $2(33.3)$ \\
\hline Missing & $5(12.5)$ & \\
\hline \multicolumn{3}{|l|}{ Length of stay in the US } \\
\hline Between 1 and 3 years & $2(5)$ & \\
\hline Between 3 and 5 years & $1(2.5)$ & Mean $=10$ years, $S D=0.816$ \\
\hline Between 5 and 7 years & $4(10)$ & \\
\hline Between 7 and 9 years & $9(22.5)$ & \\
\hline
\end{tabular}


Table 2 (continued)

\begin{tabular}{lcl}
\hline & $\begin{array}{l}\text { Survey participants } \\
(N=40) \\
f(\%)\end{array}$ & $\begin{array}{l}\text { Focus group participants }(N=6) \\
f(\%)\end{array}$ \\
\hline $\begin{array}{l}\text { 10 years or more } \\
\text { Length of stay in [current state] }\end{array}$ & $24(60)$ & \\
Less than 1 year & $3(7.5)$ & Mean $=7.75$ years, SD = 2.5 \\
Between 1 and 3 years & $7(17.5)$ & \\
Between 3 and 5 years & $4(10)$ & \\
Between 5 and 7 years & $15(37.5)$ & \\
Between 7 and 9 years & $6(15)$ & \\
10 years or more & $5(12.5)$ & \\
\hline
\end{tabular}

Fig. 2 Meaning of mental health $(n=40)$
Meaning of Mental Health among Bhutanese Refugees $(n=40)$

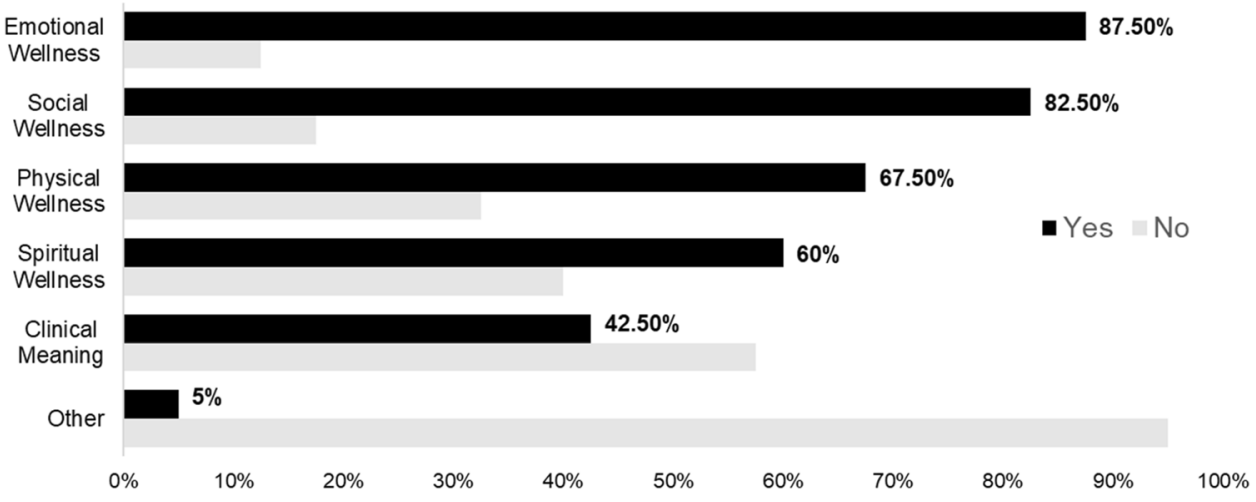

really have like a unified idea of what mental health really is. (FGD participant).

For me personally, if I wake up right in the morning-I am mentally right today. If don't wake up, right, like I have a heavy head or I'm stressed - then, I'm mentally sick for the day. So personally, I see mental health is very much attached to our personal life, day-to-day activities. (FGD participant).

In general, I don't know if there is a definition that the community has accepted or maybe still people don't know about what mental health issue is. Do I have [a] mental health issue? Or it's like it's normal as usual, I lived my life this way. (FGD participant).

Because mental health has been normalized in their daily minutiae, Bhutanese community members cannot comprehend a life free of mental health issues. FGD participants, therefore, stated that this normalcy might have contributed to the lack of awareness and understanding of mental health problems in the Bhutanese community.

\section{Theme 2: Mental Health and Culture of Inexpression}

Survey findings show widespread acknowledgment that Bhutanese community members definitely or most definitely experience mental health symptoms $(87.5 \%)$. Yet almost $32.5 \%$ of survey respondents reported that they do not experience any mental health symptoms (not at all and not likely) and $25 \%$ reported being unsure of their mental health experience (Fig. 3).

Further, self-reported anxiety measured by GAD-7 based on a mean cutoff score was mild $(M=5.67$, $\mathrm{SD}=6.32$ ) in the survey sample. Almost $55 \%$ of the survey respondents showed minimal anxiety, $20 \%$ showed mild anxiety, $12.5 \%$ showed moderate anxiety, and $12.5 \%$ showed severe anxiety levels. Similarly, depression levels, measured by PHQ-9, indicated moderately severe depression $(M=14.79, \mathrm{SD}=7.01)$ based on the cutoff score. Almost $22.5 \%$ of the survey sample showed mild depression; $42.5 \%$ showed moderate depression, $10 \%$ showed moderately severe depression, and $25 \%$ showed severe depression levels. Although survey findings suggest the prevalence of mental health symptoms in the Bhutanese community, FGD participants emphasized a lack of expression, as described by one participant: "Unexpressed. Mental health is unexpressed."

FGD participants said that the inexpression of mental health perpetuates the belief that mental health is not a relevant issue, affecting both awareness of the issue and help-seeking behavior. Participants underscored that because mental health is not expressed, there is a predominant notion that the Bhutanese community does not experience mental 
Fig. 3 Self-reported experience with mental health symptoms $(n=40)$
Experience with Mental Health Symptoms among Bhutanese Refugees $(n=40)$

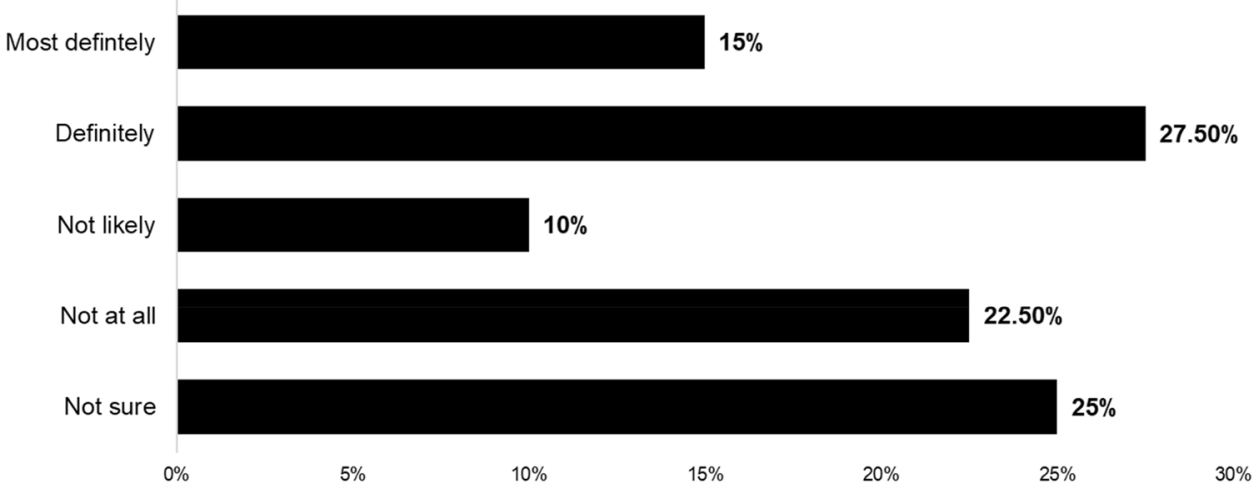

health problems and therefore, treatment is not needed for something that does not exist:

They don't consider it as an illness that has to be expressed or should seek help or support or treatment. People will say, even if they have that, the thing that I have this existing since I came or since it was like 10 years when I was in camp. So, I don't have a problem. Even if there is a problem, they don't express that. (FGD participant).

Participants corroborated several reasons for the inexpression of mental health. Bhutanese refugees adhere to a collectivist culture, wherein individual experiences are not given too much importance. Further, given their collective refugee experience, they have always had to think about safety and survival as top priorities, and expressing emotions and feelings seemed futile amid the continual disruptions they faced throughout their life course. Participants also highlighted that expression of emotional distress is limited by the Nepali language. These linguistic limitations might have contributed to the inability to express mental health experiences as understood, described, or even experienced in Western culture:

I think one of the issues is also just talking about our emotions-because we have always been on survival mode, we don't really think about ways to communicate how we feel. Also, there really isn't a way in our language to say, oh, I feel sad, or I feel isolated, because we're so into the mindset of thinking that we're constantly on survival mode and we have been, but here it's different. (FGD participant).

I don't think there are any verbiage that can define "counseling" in Nepali as of yet. So, how do people understand mental health concept in general? (FGD participant).

\section{Theme 3: Historical Trauma and Cultural Stigma}

Survey respondents (92\%) and FGD participants resoundingly agreed on the pervasive cultural stigma surrounding mental health in the Bhutanese community.

There's definitely a bad stigma, if you have mental health issues, then you're seen as like a crazy person that needs help that can't manage to do things on your own. So, it definitely has bad stigma to it. (FGD participant).

FGD participants corroborated that cultural stigma around mental health was largely due to their historical experience of collective cultural trauma that resulted in multigenerational suffering. Bhutanese refugees endured political violence and torture in Bhutan and discrimination in refugee camps in Nepal. The community's collective refugee experience of isolation and marginalization was very painful to talk about, causing repulsive reactions, which further created stigmatization of mental health. Collective cultural trauma is siphoned to subsequent generations that did not bear witness to the trauma but continue to shoulder the weight of a painfully disrupted life that inflicted emotional, psychological, and physical harm. Participants highlighted the persistent negative treatment of people suffering from mental health issues in the past that created a perceived fear of being labeled and isolated from their own community. Stigmatizing beliefs of potential violence, dangerousness, shame, incompetence, punishment, and criminality were associated with people suffering from mental illness.

What we had historically as the concept of mental health is still existing in the community. It is still taken as a source of taboo, and people don't like to talk about the painful past. They feel they will become more isolated by expressing themselves as mentally ill or mentally unable to do things. (FGD participant). 
People mostly connect mental health with some pains in the past with the family members in generations, like my uncle died by suicide. I now see that my uncle [might have] been [going through] a chronic mental health issue, but how was that taken by my family - it is like somebody in the generation before passed away - and maybe some rituals were not completed and that evil omen is still somewhere hanging around-that's how it is handled, without looking at ways to address the problem before my uncle went to that level of making the decision to take his own life. (FGD participant).

The way it was taken-back in the day-is you see people who have mental illness are being isolated. Like in my own extended family, if someone had mental illness, you are locked inside because they think they will be very violent to the family, they might do anything to the family. So, they put them in a small, closed area and they feed them once or twice a day, and that's it. So, seeing that-even if people had that mental illness, they wouldn't express that to people because of that fear. That is a historical concept of how mental health was. That is why the taboo, the stigma still exists. (FGD participant).

Participants also shared that people who experience mental health symptoms, particularly men, are seen as incapable. This cultural perception jeopardizes the traditional role and status of men in a largely patriarchal culture, in which men are expected to be strong, powerful, authoritative, and head of the household:

If you're going through some mental health issuepeople perceive them as someone who's incapable of doing and managing day-to-day lives. For us, especially the men in our community - they want to be the working person in the family_-so, like for them, these kinds of issues bother them. It's kind of putting them in a vulnerable position. So, people don't want to talk about it. (FGD participant).

\section{Theme 4: Cultural Coping Mechanisms}

FGD participants discussed positive community coping mechanisms that facilitated positive mental well-being in the Bhutanese community. Support seeking, in general, was a common coping mechanism. Survey findings on supportseeking behavior showed that it was mostly limited to the parameters of family, friends, and members of their own community (Table 3 ). The five major support-seeking behaviors were talking to people about the situation because that made them feel better $(M=1.32, \mathrm{SD}=0.77)$; describing feelings to a friend $(M=1.21, \mathrm{SD}=0.70)$; accepting help from a friend or relative $(M=1.16, \mathrm{SD}=0.78)$; telling people about the situation because it helped them come up with solutions $(M=1.13, \mathrm{SD}=0.77)$, and seeking reassurance from those who know them best $(M=1.13, \mathrm{SD}=0.81)$.

Participants in the FGD highlighted the importance of physical, mental, and spiritual practices such as yoga and walking as coping mechanisms. Bhutanese older women described household chores as a passive coping strategy to regulate their emotions:

The other thing, especially that I see very common among older women, is doing household chores-like mopping floors, cooking, cleaning, gardening, whatever it is - that really helps them take their mind off of other things. And that's something that I've noticed with my mom, even with myself right now. So, a lot of older ladies definitely use household chores as a coping mechanism. (FGD participant).

The mind-body-spirit connection was explained as significant to the Bhutanese experience. Temples and other sacred sites are considered healing spaces that provide peace and solace, not only among people experiencing mental distress but also their families. Traditional religious rituals
Table 3 Social support seeking among Bhutanese refugee population $(N=40)$

\begin{tabular}{ll}
\hline Social support items $(\alpha=0.93)$ & M (SD) \\
\hline Described your feelings to a friend & $1.21(0.7)$ \\
Accepted sympathy and understanding from someone & $1.18(0.76)$ \\
Talked to people about the situation because talking about it made you feel better & $1.32(0.77)$ \\
Talked about fears and worries to a relative or friend & $1.03(0.78)$ \\
Told people about the situation because talking about it helped you come up with solutions & $1.13(0.77)$ \\
Went to a professional to help you feel better & $0.97(0.1)$ \\
Went to a friend to help you feel better about the problem & $1.05(0.73)$ \\
Went to a friend for advice about how to change the situation & $1.08(0.71)$ \\
Accepted sympathy and understanding from friends who had the same problems & $0.95(0.73)$ \\
Accepted help from a friend or relative & $1.16(0.78)$ \\
Sought reassurance from those who know you best & $1.13(0.81)$ \\
\hline
\end{tabular}


and customs were the most commonly mentioned cultural coping mechanism. The Bhutanese community predominantly features practicing Hindus, who believe in that gods can remove the negative effects of evil powers. Because the Hindu belief system fosters concepts of collective identity, social status (i.e., varnas and caste system), ethical conduct (i.e., dharma), and familial obligations (Im \& Neff, 2020), FGD participants reiterated that family and community were very strong cultural components. Therefore, culturally supportive Hindu religious rituals—-such as "Graha Shanti," a spiritual practice to heal the spirits and the stars; collectively listening to "Bhagavad Gita," a Hindu religious scripture recited by Hindu priests; or conducting "saptaha," a sevenday prayer, worship, and ritual offering in front of a sacred fire while chanting mantras-wherein extended family members, neighbors, and the larger community are engagedprovided a sense of belonging and agency in the community. Further, participants also shared that "jhakri," who are traditional healers, have historically played a prominent role in Bhutanese culture as protectors:

In my family, if they believe there is some evil going around with any member of the family. We do religious ritual- "Graha Shanti." We invite family, where everyone come together and practice these religious and spiritual practices. (FGD participant).

If someone has mental illness, they still believe that going to temple solves that problem. Because back home, they used to go Brindaban [religious site]. So similarly, here also they go to pilgrims, there is "saptaha" a religious ceremony that is being conducted that runs for seven days. A religious event is being set up by the family where the immediate family member sits in an isolated place and listen to the Bhagavad Gita recited by the pundits [priests]. We still see some old kind of practice of using "dhami-jhakiri." (FGD participant).

Younger FGD participants emphasized the use of technology and social media to share their thoughts and feelings with their Bhutanese peers. Participants highlighted the lack of Nepali-speaking counselors and counselors of color as a barrier to seeking mental health services, but they could find support on online platforms. Bhutanese refugee youth utilized digital technology such as FaceTime and social media platforms such as Facebook private groups, Finstagram (a private Instagram space or account), Twitter, and TikTok to express emotions and share everyday difficulties of coping with cultural demands, academic difficulties, and cultural conflicts. Younger FGD participants affirmed that there is a widespread acknowledgement and acceptance of mental health in this demographic group, and that these digital spaces are convenient mediums to not only talk about mental health, but also curate actionable steps for healing, maintain social relationships, and help promote a system of support and sense of community.

I think for my generation, we understand the concept of mental health. But it's more so, like, how do we deal with it? And we don't have counselors who speak Nepali and it's usually hard to get a counselor of color. So, for us, it's like, what are some things that we could do to cope, and one of them has just been talking to each other via Facebook, FaceTime. But I do think that we need to kind of talk about ways to heal also. What are some steps that we could take or what are some things that we can learn to help? (FGD participant). There's a private Instagram—called Finsta-where you just rant about what's going on and you only have a selected group of friends that can see it. Rather than calling each friend, I can tell them what's going on with my life. And some do give feedback. Other times, I just need to vent or say something. I've also seen people post a lot of emotional hardship on Twitter. TikTok is also becoming a big thing, where people are complaining about daily things that they go through in life, especially about cultural differences. So, there are various different platforms that my age group have been using to cope with what's going on, to show they're not alone in this. We have built some type of community. (FGD participant).

FGD participants described the important role of community-based initiatives and support groups such as soccer teams, youth support groups, arts and literature groups, and book clubs to navigate mental health stressors in the Bhutanese community. Younger FGD participants highlighted the impactful work of peer-led groups, such as Women of Knowledge and Empowerment (WOKE), to create a platform to talk about mental health issues. WOKE was essentially started by young Bhutanese women who participated in a 12-month cultural leadership project (Maleku et al., 2018), an academic-community partnership project organized to promote cultural identity and leadership among young Bhutanese refugee women. Participants shared that the enriching discussions that take place in these spaces were beneficial for both facilitators and participants alike.

For our generation, we have the WOKE and the soccer team. One of the goals of the group is to also tackle mental health and talk about it. So, those groups have been very intimate for us at this point. And we really share some of the things that we have been going through. Just nonverbal comments that we receive in the group have been very helpful. For the younger girls and us as facilitators, we are actually getting benefits from that group. (FGD participant). 
FGD participants discussed the role of arts and literature among Bhutanese community members and highlighted how expressive writing through literature groups were used as coping mechanisms to ease stress. Expressive writing in the form of poems, literary pieces, stories, songs, and books gave meaning to ruminating thoughts, experiences of trauma, and emotional distress. Participants highlighted that these literary groups provided a pathway for social connection and community healing. Further, the festive feel to the literary meetings - where community members presented themselves in ethnic cultural attire-promoted cultural identity in the Bhutanese refugee population.

There is also arts and literate. There is a literary group-it's a group where they-[people] who can read and write Nepali-form a club, where they write poetry, wear traditional outfits, write sort of stories and things like that, and publish book. Because that is also another way of sharing their experiences. I've seen lots of people are writing songs, making albums, and sharing that. These are ways of sharing expression of what their emotions are. We have bunch of youth clubs where they are now starting to organize book clubs. (FGD participant).

In addition to these positive coping mechanisms, FGD participants also shared negative coping mechanisms that are pervasive in the Bhutanese community. From rising rates of alcohol and substance use to gambling and alarming rates of suicide, participants affirmed that these negative coping strategies were a byproduct of the cultural stigma associated with mental illness and negative attitudes toward people suffering from emotional distress, which create layers of suppressed emotions until it erupts into severe repercussions:

I think so many people are going negatively-like getting addicted to alcohol and drugs, gambling, and one of the worst-case scenarios is the suicide rate, which is very high in the community. It cannot be expressed, I still believe for somebody like me and in the generation older to us, if you express it, you are seen as a lunatic, like you are crazy. So, it remains unexpressed like a magma and people get distracted completelythey take negatives routes like addiction and alcoholism-and you feel like taking your own life. (FGD participant).

\section{Discussion and Implications}

Findings show that the Bhutanese community's loose understanding of mental health is tied to traumatic historical experiences, which affects cultural stigma around the issue of mental health and those who experience it. We found that the collective mental health experience of the Bhutanese refugee community remains largely unexpressed and unaddressed, which further contributes to the stigma associated with mental health. In our study, Bhutanese refugees' collective survival; traumatic history of violence, discrimination, and marginalization; and collective normalization of emotional distress in daily life contributed to mental health inexpression (Sangalang \& Vang, 2017). These findings corroborate previous studies that have shown that Bhutanese refugees find it unhelpful to share their suffering with community members sharing collective trauma (Shannon et al., 2015b). Consistent with previous studies, we found that negative experiences with hospitalization and treatment and fears of being labelled, shamed, and isolated from their community contributed to inexpression of mental health symptoms (MacDowell et al., 2020). These fears stem from a history of political repression, perpetual threats, and disconnection and disempowerment in new spaces that leave refugees helpless and speechless (Shannon et al., 2015a, b). Even after achieving safety in places of resettlement, refugees are less likely to speak freely about their past and current suffering due to decades of silent survival (Shannon et al., 2015a). Findings show the strong influence of culture on mental health inexpression. The value placed on thoughtful and self-disciplined silence in collective cultures (Kim \& Sherman, 2007) disinclines Bhutanese from expressing mental distress. Further, the Nepali language is limited in terminologies to describe mental health. These findings point to the link between mental health and somatization (Lanzara et al., 2019) in the Bhutanese refugee population, largely due to differences in expressing emotional distress, limited by their ethnic cultural language. Often, cultures are made visible through languages. Scholars have long pointed to the difficulties of accurately describing experiences that involve the mind and body across different cultures. Further efforts to address emotional distress - with special attention to social, cultural, and linguistic differences-are therefore warranted for the overall mental well-being of all refugees (Lanzara et al., 2019). More in-depth studies on local conceptualization of mind and body and expression through language in the Bhutanese refugee population are much needed to explore unique cultural and linguistic contexts of mental health and somatization (Kohrt \& Hruschka, 2010). Future studies could use inductive approaches to provide more in-depth understanding of cultural vernacular and idioms of distress in the Bhutanese refugee population to broaden our understanding of mental health expression and communication.

Study findings show that recognition and acceptance of mental health were more widespread among Bhutanese youth compared to older generations. Further, their use of digital technology - as coping mechanism and as a way to find collective healing-points to the potential for targeted 
digital mental health applications and use of digital platforms for Bhutanese refugee youth. Our study contributes to the critical need to explore mental health challenges across demographic subgroups of Bhutanese refugees, especially youth (Betancourt et al., 2020). Findings on the Bhutanese community's negative and positive cultural coping strategies provide implications for targeted programs and services. Study findings allude to negative coping mechanisms such as alcohol, substance use, suicidal ideation, and suicide attempts, consistent with prior studies (Adhikari et al., 2015; Michaels et al., 2010; Mirza et al., 2018). Bhutanese refugees feel more comfortable seeking help from friends and family than from professionals (Chase \& Sapkota, 2017), consistent with prior studies showing that this community generally views care seeking as negative and a sign of weakness (MacDowell et al., 2020). Study findings suggest that religious and spiritual practices were positive coping mechanisms. Findings from our study show that Hindu rituals and practices are central to cultural coping (Chase et al., 2013); engaging in culturally supportive rituals helped promote mental well-being (Calabrese \& Dorji, 2013); and religious and spiritual affiliation provided a sense of belonging and agency (Amit \& Bar-Lev, 2015; Benson et al., 2012). Findings show that community-based initiatives formed around Bhutanese arts and literature, women's support groups, youth clubs, and sports have been used as mechanisms to bring the community together based on shared identity and experiences. Because refugee populations rely more on their social networks, community-based initiatives can provide collective platforms for cultural healing that promote positive mental well-being. Because culture is a significant coping mechanism that builds resilience, these identified cultural ways of coping are crucial protective factors that can minimize risk factors for the overall psychosocial well-being of Bhutanese refugees (Pulla, 2016). We argue that recognizing the historical context and cumulative migration stressors that contribute to present-day psychological distress is paramount to cultural healing and resilience in subsequent generations. Given the collective historical experience with trauma, development of culturally tailored interventions that go beyond treatment of symptoms to overall healing and well-being are necessary to effectively address mental health inequities experienced by the Bhutanese refugee population. Understanding how historical trauma might influence the current mental health status of the ethnic Bhutanese refugee population and attention to issues of somatization, language and expression of emotional distress may provide new directions for eliminating mental health inequities in this population. Given the collective culture, mental health education and intervention should be targeted at the community level, using culturally grounded initiatives that already exist in the community. Using cultural assets of the community will have a far-reaching impact on collective healing.

\section{Limitations}

Our study has several limitations. Given the small sample size, any attempts to make statistical inferences or generalize the study findings outside the Midwestern geographic region might be inconclusive. Our study was limited in examining gender differences in expression of emotional distress and coping responses. The nature of the study exploring the sensitive topic of mental health was itself a limitation for sample recruitment, because many participants did not want to talk about it. This could have also led to higher representation of the younger age group in the survey sample, because they were more accepting of the issue. There were challenges with data collection process due to language barriers, lower literacy levels of participants, and the disruption caused by COVID-19. Given the virtual adjustment of the FGD due to COVID-19 disruption, community participation might have been limited, largely due to uneven access to broadband internet or technology. Although translators were used to offset some of the language and literacy limitations, this could have led to potential self-reporting biases. Further, data collection at the community-based organization mostly occurred in an open setting, which might have played a role in response biases. The FGD was conducted in both English and Nepali, transcribed verbatim, and translated into English. Although verbatim responses have been used to maintain data originality, it is possible that data could have been misinterpreted or the original meanings might have been lost in translation. The use of standardized scales such as PHQ-9 and GAD-7, although used across refugee populations, adapted, and used among Nepali-speaking populations, might have been limited in capturing the unique Bhutanese refugee context. Despite these limitations, however, our study captured a multiplicity of community voices to elucidate the conceptualization of mental health through the Bhutanese refugee lens. As such, findings are well positioned to inform culturally responsive community mental health services and provide implications for diversity and inclusion efforts in local and regional contexts.

\section{Conclusion}

Our study provided a multidimensional conceptualization of mental health through the Bhutanese refugee lens based on unique historical and cultural contexts, crucial for devising culturally responsive mental health services and interventions. Collective histories of trauma shared by communities can result in cumulative emotional and psychological impacts that linger across generations. The persistent cycle of trauma not only weakens families and communities, but also deteriorates the vibrancy of cultures. Our study contributed to knowledge around community coping mechanisms at 
the intersection of religion, spirituality, cultural identity, and digital technology. Acknowledging the processes of underlying intergenerational trauma in community mental health services can bolster cultural healing. Holistic interventions that target culturally based collective healing and not just symptoms of mental health are crucial to bolstering resilience in this population. Further, participatory approaches that include the Bhutanese community will help create knowledge and community-based, healing-centered interventions to best serve these new members of U.S. society. We hope our study will spark additional research to better understand this unique refugee population facing a mental health crisis while simultaneously offering culturally grounded directions to promote mental health equity in this population.

Acknowledgements The authors would like to thank the Bhutanese community organization that helped with the implementation of the study.

\section{Declaration}

Conflict of interest The authors declare that they have no conflict of interest.

\section{References}

Amit, K., \& Bar-Lev, S. (2015). Immigrants' sense of belonging to the host country: The role of life satisfaction, language proficiency, and religious motives. Social Indicators Research, 124(3), 947-961

Ao, T., Shetty, S., Sivilli, T., Blanton, C., Ellis, H., Geltman, P. L., Cochran, J., Taylor, E., Lankau, E. W., \& Cardozo, B. L. (2016). Suicidal ideation and mental health of Bhutanese refugees in the United States. Journal of Immigrant and Minority Health, 18(4), $828-835$

Baranik, L. E., Hurst, C. S., \& Eby, L. T. (2018). The stigma of being a refugee: A mixed-method study of refugees' experiences of vocational stress. Journal of Vocational Behavior, 105, 116-130

Benson, G. O., Sun, F., Hodge, D. R., \& Androff, D. K. (2012). Religious coping and acculturation stress among Hindu Bhutanese: A study of newly-resettled refugees in the United States. International Social Work, 55(4), 538-553

Betancourt, T. S., Berent, J. M., Freeman, J., Frounfelker, R. L., Brennan, R. T., Abdi, S., Maalim, A., Abdi, A., Mishra, T., Gautam, B. Creswell, J. W. \& Beardslee, W. R. (2020). Family-based mental health promotion for somali bantu and bhutanese refugees: Feasibility and acceptability trial. Journal of Adolescent Health, 66(3), 336-344

Betancourt, T. S., Frounfelker, R., Mishra, T., Hussein, A., \& Falzarano, R. (2015). Addressing health disparities in the mental health of refugee children and adolescents through communitybased participatory research: A study in 2 communities. American Journal of Public Health, 105(S3), S475-S482

Bjarta, A., Leiler, A., Ekdahl, N., \& Wasteson, E. (2018). Assessing severity of psychological distress among refugees with the refugee health screener, 13-item version. The Journal of Nervous and Mental Disease, 206(11), 834-839
Bonevski, B., Randell, M., Paul, C., Chapman, K., Twyman, L., Bryant, J., Brozek, I., \& Hughes, C. (2014). Reaching the hard-to-reach: A systematic review of strategies for improving health and medical research with socially disadvantaged groups. Medical Research Methodology, 14(42), 1-29

Calabrese, J. D., \& Dorji, C. (2013). Traditional and modern understandings of mental illness in Bhutan: Preserving the benefits of each to support gross national happiness. Journal of Bhutan Studies, 30, 1-29

Chase, L., \& Sapkota, R. P. (2017). "In our community, a friend is a psychologist": An ethnographic study of informal care in two Bhutanese refugee communities. Transcultural Psychiatry, 54(3), 400-422

Chase, L. E., Welton Mitchell, C., \& Bhattarai, S. (2013). Solving tension: Coping among Bhutanese refugees in Nepal. International Journal of Migration, Health and Social Care, 9(2), 71-83

Cochran, J., Geltman, P. L., Ellis, H., Brown, C., Anderton, S., Montour, J., Vargas, M., Komatsu, K., Senseman, C., Lopes Cardozo, B., Sivilli, T. I., Blanton, C., Shetty, S., Taylor, E., Lankau, E., $\&$ Ao, T. (2013). Suicide and suicidal ideation among Bhutanese refugees-United States, 2009-2012. Morbidity and Mortality Weekly Report, 62(26), 533-536

Collins, K. M. T., Onwuegbuzie, A. J. \& Jiao, Q. G. (2007). A mixed methods investigation of mixed methods sampling designs in social and health science research. Journal of Mixed Methods Research, 1(3), 267-294

Cramer, R. J., \& Kapusta, N. D. (2017). A socio-ecological framework of theory, assessment, and prevention of suicide. Frontiers in Psychology, 8, 1756

Creamer, E. (2018). An introduction to fully integrated mixed methods research. Thousand Oaks: Sage.

Creswell, J. W. (2015). A concise introduction to mixed methods research. Thousand Oaks: Sage.

Crosby, R. A. Salazar, L. F. DiClemente, R. J. \& Lang, D. L. (2010). Balancing rigor against the inherent limitations of investigating hard-to-reach populations. Health Education Research, 25(1), 1-5

Derr, A. S. (2016). Mental health service use among immigrants in the United States: A systematic review. Psychiatric Services, 67(3), 265-274

Desmond, D. M., Shevlin, M. \& MacLachlan, M. (2006). Dimensional analysis of the coping strategy indicator in a sample of elderly veterans with acquired limb amputations. Personality and Individual Differences, 40(2), 249-259

Ehlers, C. L., Gizer, I. R., Gilder, D. A., Ellingson, J. M., \& Yehuda, R. (2013). Measuring historical trauma in an American Indian community sample: Contributions of substance dependence, affective disorder, conduct disorder and PTSD. Drug and Alcohol Dependence, 133(1), 1-21

Ellis, B. H., Hulland, E. N., Miller, A. B., Bixby, C. B., Cardozo, B. L., \& Betancourt, T. S. (2016). Mental health risks and resilience among Somali and Bhutanese refugee parents. Migration Policy Institute. https://www.migrationpolicy.org/sites/default/ files/publications/FCD-Ellis-FINAL.pdf

Evans, R. (2010). The perils of being a borderland people: On the Lhotshampas of Bhutan. Contemporary South Asia, 18(1), 25-42. https://doi.org/10.1080/09584930903561598

Fetters, M. D., Curry, L. A. \& Creswell, J. W. (2013). Achieving integration in mixed methods designs- principles and practices. Health Services Research, 48(6 Pt 2), 2134-2156

Gupta, A. K., Mehra, A., Niraula, A., Kafl, K., Deo, S. P., Singh, B., Sahoo, S., \& Grover, S. (2020). Prevalence of anxiety and depression among the healthcare workers in Nepal during the COVID-19. Asian Journal of Psychiatry, 54, 102260

Gurung, N., \& Baidya, P. (2010, August 9). A fresh start: Refugees from Bhutan arrive in the UK. United Nations High 
Commissioner for Refugees. http://www.unhcr.org/print/4c602 6059.html

Hacker, K. (2013). Community-based participatory research. Sage Publications Inc

Hagaman, A. K., Sivilli, T. I., Ao, T., Blanton, C., Ellis, H., Cardozo, B. L., \& Shetty, S. (2016). An investigation into suicides among Bhutanese refugees resettled in the United States between 2008 and 2011. Journal of Immigrant and Minority Health, 18(4), 819-827

Heart, M. Y. H. B. (2003). The historical trauma response among natives and its relationship with substance abuse: A lakota illustration. Journal of Psychoactive Drugs, 35(1), 7-13. https://doi. org/10.1080/02791072.2003.10399988

Hennink, M. M., Kaiser, B .N. \& Weber, M. B. (2019). What influences saturation? Estimating sample sizes in focus group research. Qualitative Health Research, 29(10), 1483-1496

Im, H., Ferguson, A., \& Hunter, M. (2017). Cultural translation of refugee trauma: Cultural idioms of distress among Somali refugees in displacement. Transcultural Psychiatry, 54(5-6), 626-652

Im, H., \& Neff, J. (2020). Spiral loss of culture: Cultural trauma and bereavement of Bhutanese refugee elders. Journal of Immigrant \& Refugee Studies. https://doi.org/10.1080/15562948.2020.1736362

Ivankova, N. V., Creswell, J. W., \& Stick, S. L. (2006). Using mixedmethods sequential explanatory design: From theory to practice. Field Methods, 18(1), 3-20

Kim, H. S., \& Sherman, D. K. (2007). "Express yourself": Culture and the effect of self-expression on choice. Journal of Personality and Social Psychology, 92, 1-11

Kohrt, B. A., \& Hruschka, D. J. (2010). Nepali concepts of psychological trauma: The role of idioms of distress, ethnopsychology and ethnophysiology in alleviating suffering and preventing stigma. Culture, Medicine, and Psychiatry, 34(2), 322-352

Kohrt, B. A., Kunz, R. D., Baldwin, J. L., Koirala, N. R., Sharma, V. D., \& Nepal, M. K. (2005). "Somatization" and "comorbidity": A study of Jhum-Jhum and depression in rural Nepal. Ethos, 33(1), 125-147

Kohrt, B. A., Luitel, N. P., Acharya, P., \& Jordans, M. J. (2016). Detection of depression in low resource settings: Validation of the Patient Health Questionnaire (PHQ-9) and cultural concepts of distress in Nepal. BMC Psychiatry, 16, 58

Kroenke, K., Spitzer, R. L., \& Williams, J. B. (2001). The PHQ-9: Validity of a brief depression severity measure. Journal of General Internal Medicine, 16(9), 606-613

Lanzara, R., Scipioni, M., \& Conti, C. (2019). A clinical-psychological perspective on somatization among immigrants: A systematic review. Frontiers in Psychology, 9, 2792

Maack, B., \& Willborn, R. (2018). Description of tobacco use and cessation among Bhutanese refugees participating in a tobacco cessation program. Ethnicity \& Health, 23(4), 367-379

MacDowell, H., Pyakurel, S., Acharya, J., Morrison-Beedy, D., \& Kue, J. (2020). Perceptions toward mental illness and seeking psychological help among Bhutanese refugees resettled in the US. Issues in Mental Health Nursing, 41(3), 243-250

Maleku, A., \& Aguirre, R. (2014). Culturally competent healthcare from the immigrant lens: A qualitative interpretive meta-synthesis. Social Work in Public Health, 29(6), 561-580

Maleku, A., Kagotho, N., Karandikar, S., Mengo, C., Freisthler, B., Baaklini, V., \& Filbrun, C. (2018). The new Americans project: Assessing the human service landscape in central Ohio. Columbus, Ohio: The Ohio State University College of Social Work

McHugh, M. L. (2012). Interrater reliability: The kappa statistic. Biochemia Medica (Zagreb), 22(3), 276-282

Michaels, C., Rousseau, R., \& Yang, Y. (2010). Historical trauma and microaggressions: A framework for culturally-based practice. Children's Mental Health eReview, 1-9.

Mirza, M. Q., Harrison, E. A., Chang, H. C., Salo, C. D., \& Birman, D. (2018). Community perspectives on substance use among
Bhutanese and Iraqi refugees resettled in the United States. Journal of Prevention \& Intervention in the Community, 46(1), 43-60.

Padgett, D. K. (2017). Qualitative methods in social work research. 3rd Edition. Sage Publications Inc

Porter, M., \& Haslam, N. (2003). Predisplacement and postdisplacement factors associated with mental health of refugees and internally displaced persons: A meta-analysis. Journal of the American Medical Association, 294, 602-612

Poudel-Tandukar, K., Jacelon, C. S., Chandler, G. E., Gautam, B., \& Palmer, P. H. (2019). Sociocultural perceptions and enablers to seeking mental health support among Bhutanese refugees in Western Massachusetts. International Quarterly of Community Health Education, 39(3), 135-145

Pulla, V. (Ed.). (2016). The Lhotsampa people of Bhutan: Resilience and Survival. New York: Palgrave Macmillan

Refugee Processing Center. (2020). Interactive reporting: Admissions and arrivals. https://www.wrapsnet.org/

Rinker, J. A., \& Khadka, N. (2018). Bhutanese refugees: On understanding the links between trauma, displacement, and community resilience. Global Journal of Peace Research and Praxis, 2(1), $1-19$

Ritsner, M., Ponizovsky, A., Kurs, R., \& Modai, I. (2000). Somatization in an immigrant population in Israel: A community survey of prevalence, risk factors, and help-seeking behavior. American Journal of Psychiatry, 157, 285-392

Rohlof, H. G., Knipscheer, J. W., \& Kleber, R. J. (2014). Somatization in refugees: A review. Social Psychiatry Psychiatric Epidemiology, 49(11), 1793-1804. https://doi.org/10.1007/ s00127-014-0877-1

Saldana, J. (2016). The coding manual for qualitative researchers Thousand Oaks: Sage.

Sangalang, C. C., \& Vang, C. (2017). Intergenerational trauma in refugee families: A systematic review. Journal of Immigrant and Minority Health, 19(3), 745-754

Schininà, G., Sharma, S., Gorbacheva, O., \& Mishra, A. K. (2011). Who am I? Assessment of psychosocial needs and suicide risk factors among Bhutanese refugees in Nepal and after the third country resettlement. International Organization for Migration. https://www.iom.int/files/live/sites/iom/files/What-We-Do/docs/ Mental-Health-Assessment-Nepal_Final_11March.pdf

Shaghaghi, A., Bhopal, R. S., \& Sheikh, A. (2011). Approaches to recruiting "hard-to-reach" populations into research: A review of the literature. Health Promotion Perspectives, 1(2), 86-94

Shannon, P. J., Wieling, E., Simmelink-McCleary, J., \& Becher, E. (2015a). Beyond stigma: Barriers to discussing mental health in refugee populations. Journal of Loss and Trauma, 20(3), 281-296

Shannon, P. J., Wieling, E., Simmelink-McCleary, J., \& Becher, E. (2015b). Exploring the mental health effects of political trauma with newly arrived refugees. Qualitative Health Research, 25(4), 443-457. https://doi.org/10.1177/1049732314549475

Shrestha, D. D. (2015, November 19). Resettlement of Bhutanese refugees surpasses 100,000 mark. United Nations High Commissioner for Refugees. http://www.unhcr.org/564dded46.html

Sotero, M. (2006). A conceptual model of historical trauma: Implications for Public Health Practice and Research. Journal of Health Disparities Research and Practice, 1(1), 93-108

Spitzer, R. L., Kroenke, K., Williams, J. B., \& Löwe, B. (2006). A brief measure for assessing generalized anxiety disorder. Archives of Internal Medicine, 166(10), 1092-1097

Staples, J., \& Widger, T. (2012). Situating suicide as an anthropological problem: ethnographic approaches to understanding self-harm and self-inflicted death. Culture, Medicine and Psychiatry, 36(2), 183-203

Tcholakian, L. A., Khapova, S. N., Loo, E., \& Lehman, R. (2019). Collective traumas and the development of leader values: A currently 
omitted, but increasingly urgent, research area. Frontiers in Psychology, 10, 1009

Teddlie, C., \& Tashakkori, A. (2009). Foundations of mixed methods research: Integrating qualitative and quantitative approaches in the social and behavioral sciences. Thousand Oaks: Sage.

Teunissen, E., Sherally, J., van den Muijsenbergh, M., Dowrick, C., van Weel-Baumgarten, E., \& Van Weel, C. (2014). Mental health problems of undocumented migrants (UMs) in the Netherlands: A qualitative exploration of help-seeking behaviour and experiences with primary care. BMJ Open, 4(11), e005738

Tolan, C. (2016, June 9). A mysterious mental health disorder is afflicting Bhutanese refugees in America. Splinter. https://splinterne ws.com/a-mysterious-mental-health-disorder-is-afflicting-bhuta1793857392

U.S. Department of State. (2017). Proposed refugee admissions for fiscal year 2017: Report to the Congress. https://2009-2017.state. gov/j/prm/releases/docsforcongress/261956.htm

van der Boor, C. F., \& White, R. (2020). Barriers to accessing and negotiating mental health services in asylum seeking and refugee populations: The application of the candidacy framework. Journal of Immigrant Minority Health, 22, 156-174

Van Ommeren, M., de Jong, J. T. V. M., Sharma, B., Komproe, I., Thapa, S. B., \& Cardeña, E. (2001). Psychiatric disorders among tortured Bhutanese refugees in Nepal. Archives of General Psychiatry, 58, 475-482
Vonnahme, L. A., Lankau, E. W., Ao, T., Shetty, S., \& Cardozo, B. L. (2015). Factors associated with symptoms of depression among Bhutanese refugees in the United States. Journal of Immigrant and Minority Health, 17(6), 1705-1714

Watkins, D. C. (2017). Rapid and rigorous qualitative data analysis: The "RADaR" technique for applied research. International Journal of Qualitative Methods. https://doi.org/10.1177/1609406917 712131

Watkins, D. C., \& Gioia, D. (2015). Mixed methods research. New York: Oxford University Press.

Wylie, L., Meyel, R. V., Harder, H., Sukhra, J., Luc, C., Ganjavi, H., Elfakhani, M., \& Wardrop, N. (2018). Assessing trauma in a transcultural context: Challenges in mental health care with immigrants and refugees. Public Health Reviews, 39, 22

Yakushko, O., Watson, M., \& Thompson, S. (2008). Stress and coping in the lives of recent immigrants and refugees: Considerations for counseling. International Journal for the Advancement of Counselling, 30, 167-178

Publisher's Note Springer Nature remains neutral with regard to jurisdictional claims in published maps and institutional affiliations. 